\title{
1 Low oxygen arrests Babesia duncani schizonts and leads to increased \\ 2 drug susceptibilities in hamster erythrocytes
}

3 Yumin Zhang ${ }^{\mathrm{a}}$, Hector Alvarez-Manzo ${ }^{\mathrm{a}}$, and Ying Zhang ${ }^{\mathrm{a}, \mathrm{b}^{*}}$

$4 \quad{ }^{\mathrm{a}}$ Department of Molecular Microbiology and Immunology, Johns Hopkins Bloomberg

5 School of Public Health, Johns Hopkins University, Baltimore, MD, USA

$6 \quad{ }^{\mathrm{b}}$ State Key Laboratory for the Diagnosis and Treatment of Infectious Diseases, National

7 Clinical Research Center for Infectious Diseases, The First Affiliated Hospital, Zhejiang

8 University School of Medicine, Hangzhou 310003, China

9 *Correspondence: Ying Zhang, MD, PhD, Email: medlife207@ yahoo.com

\section{Abstract}

12 We studied the effect of oxygen concentrations on the in vitro growth and drug

13 susceptibility of Babesia duncani. We found that the growth of B. duncani required high

14 level oxygen and the culture condition at ambient aerobic condition $\left(21 \% \mathrm{O}_{2}\right)$ was

15 optimal. Compared with ambient air, our results further showed that low oxygen (6-

$1616 \%$ ) could arrest B. duncani schizonts and lead to high susceptibilities to antiparasitic

17 drugs atovaquone, pyrimethamine, quinine, and chloroquine at certain concentrations in

18 vitro. Drug susceptibilities of other Babesia spp impacted by $\mathrm{O}_{2}$ levels need to be

19 studied in the future, and this study indicates that culturing conditions of Babesia spp

20 should be considered and reestablished for generating more comparable and reliable

21 results in drug research in the future.

23 Keywords: Babesia duncani, oxygen, drug susceptibility 


\section{Graphical abstract}

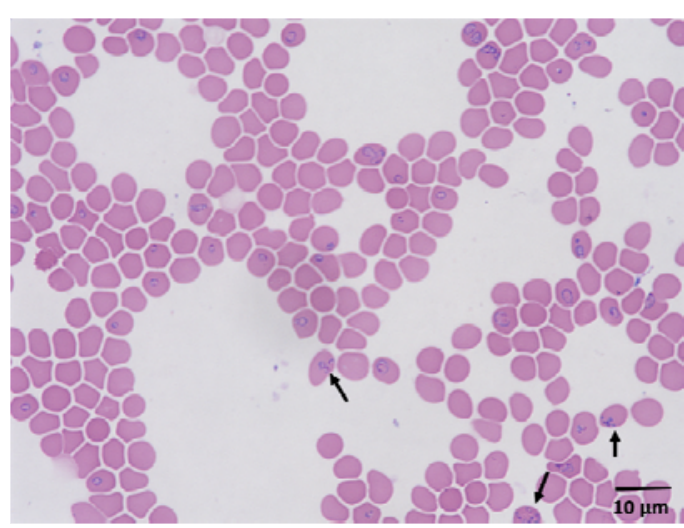

Morpholosy of Babeska duncanl cultured in 21\% $\mathrm{O}_{2}$

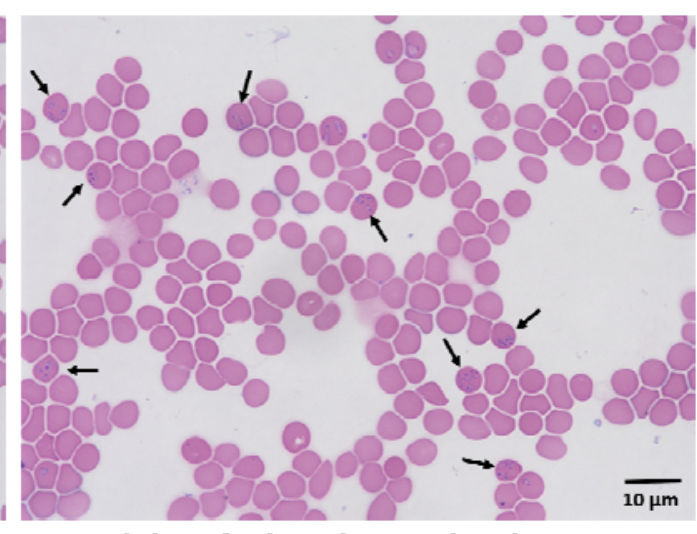

Morphology of Babesla dencowl cultured in 6-16\% $\mathrm{O}_{2}$

28 Highlights

- The in vitro growth of $B$. duncani requires high level oxygen and the culture condition of $5 \% \mathrm{CO}_{2}$ ambient air is optimal

- Low level oxygen results B. duncani in low growth rate and high schizont ratio in vitro

- Compared with $5 \% \mathrm{CO}_{2}$ ambient air, in vitro drug susceptibilities of B. duncani can be significantly increased when cultured in microaerobic condition 
37 Babesiosis is one of the most important tick-borne infectious diseases in Europe and

38 North America and has been added to the list of nationally notifiable disease by the

39 Centers for Disease Control and Prevention (CDC) of the United States . The clinical

40 symptoms of babesiosis patients range from flu-like mild to malaria-like severe

41 manifestations and can even cause death in elderly and immunocompromised patients.

42 However, the current therapeutic regimen to human babesiosis is suboptimal with

43 significant side effects and occasionally recrudescence (Kjemtrup and Conrad, 2000).

44 For development of more effective treatments, continuous in vitro culture of

45 Babesia spp is one of the most critical techniques to explore active drugs. In the past

46 decade, dozens of promising anti-babesia compounds have been identified with this

47 technique. The first attempt to establish in vitro cultivation of Babesia spp in any real

48 sense was in 1980, when Timms cultivated B. bovis, B. bigemina, and B. rodhaini in

49 vitro up to $96 \mathrm{~h}$ using the candle jar technique which mimicked the in vitro culture of

50 Plasmodium falciparum by Trager and Janson (Jensen and Trager, 1977; Timms, 1980).

51 Subsequently, Levy and Ristic successfully carried out continuous cultivation of $B$.

52 bovis in a stationary microaerophilic phase where oxygen had decreased to the level that

53 could darken red blood cells laid on plate bottom (Levy and Ristic, 1980). In 1985,

54 Vega et al. further demonstrated that high oxygen (5.6\% - normal air) is detrimental to

55 in vitro growth of B. bigemina (Vega et al., 1985). Thus, it seems that Babesia spp are

56 microaerophilic organisms like P. falciparum. However, the in vitro cultivations of two

57 important human babesiosis causative agents $B$. divergens and $B$. duncani were set in

58 different $\mathrm{O}_{2}$ levels in some studies for drug activity testing or drug screening (Abraham

59 et al., 2018; Brasseur et al., 1998; Nehrbass-Stuedli et al., 2011; Zhang et al., 2020),

60 which have could lead to divergent results in anti-malarial drug studies (Branco et al.,

61 2018; Duffy and Avery, 2018). Thus, two interesting questions are raised here: first, 
62 does B. duncani grow well in low $\mathrm{O}_{2}$; second, is $\mathrm{O}_{2}$ level a factor that affects the anti-

63 babesia drug activity in vitro?

64 To address these questions, here we studied the effects of $\mathrm{O}_{2}$ levels on the

65 growth and drug tolerance of B. duncani (WA1 strain), the latest human babesiosis

66 pathogen to be identified. In continuous culture model in hamster erythrocytes

67 cultivated in $5 \% \mathrm{CO}_{2}$ ambient air with humidity (Zhang et al., 2020), we found $B$.

68 duncani schizont was the major morphology in old cultures (5-6 culture days, $0.5 \%$ of

69 initial parasitemia) and this morphology rarely appeared in young cultures (Figure 1A).

70 These schizonts appearing as budding four daughter merozoites in erythrocytes and

71 displayed several different scattered distributions (Figure 1, B1-B6). Considering

72 culture medium changed daily and erythrocytes turned dark in old cultures, we

73 hypothesize that low oxygen environment resulting from rapid oxygen consumption by

74 parasites in high parasitemia is related to the increasing percentage of schizonts. To

75 confirm this, when parasitemia reached to $1.5-2 \%$ cultivated in $5 \% \mathrm{CO}_{2}$ atmosphere, we

76 split the B. duncani cultures into anaerobic, microaerobic, and aerobic conditions

77 without other culturing condition change to evaluate the effects of oxygen

78 concentrations on parasite growth and morphologic development. In this study,

79 anaerobic $\left(0.7-1 \% \mathrm{O}_{2}\right)$ and microaerobic $\left(6-16 \% \mathrm{O}_{2}\right)$ conditions were generated by BD

80 GasPak EZ container systems with CampyPak container system sachets and anaerobe

81 container system sachets, respectively, and $5 \% \mathrm{CO}_{2}$ atmosphere was viewed as aerobic

82 condition $\left(21 \% \mathrm{O}_{2}\right.$, approximately). Even though the final $\mathrm{CO}_{2}$ concentrations in $\mathrm{BD}$

83 GasPak EZ container systems would be various from $2 \%$ to $13 \%$ according to the

84 manufactory's instructions, our results indicated that the rate of growth and

85 morphologic development of B. duncani did not show significant difference among 2, 5,

86 and $13 \% \mathrm{CO}_{2}$ atmosphere air the cultures incubated in, but B. duncani did not grow in 
87 atmosphere air deprived $\mathrm{CO}_{2}$ (data not shown). Culture medium covered erythrocytes

88 replaced every day with fresh medium which had been co-incubated in respective

89 culture environments to minimize the impact of the fluctuation of nutrition and

90 dissolving oxygen.

Our results showed that the growth of $B$. duncani incubated in microaerophilic

92 condition reached to peak parasitemia of approximate $16 \%$ at day 5 . As a comparison,

93 the parasitemia of cultures incubated in aerobic condition could finally reach to

94 approximate $40 \%$ at day 5 before erythrocyte lysis, and it grew faster than that in

95 microaerophilic condition after 1 day (Figure 2). Growth of B. duncani stopped when

96 incubated in anaerobic condition, and no infected erythrocyte was observed starting at

97 day 3 (Figure 2). This indicates that B. duncani is an oxygen preferred organism, and

98 the growth of B. duncani requires more oxygen than only $2-5 \% \mathrm{O}_{2}$ employed in

99 previous studies which may not be the optimal in vitro growth condition for $B$. duncani

100 (Abraham et al., 2018; McCormack et al., 2019). It can partly explain why the $B$.

101 duncani cultures obtained in ambient air was able to reach to much higher parasitemia

102 than reported parasitemias, i.e., $40 \%$ in our study vs $20 \%$ or less previously (Abraham

103 et al., 2018; McCormack et al., 2019). Of note, the pericellular $\mathrm{O}_{2}$ around the parasites

104 and erythrocytes laid on plate bottom must be lower than environmental $\mathrm{O}_{2}$ due to the

105 culture medium with depth of $0.7 \mathrm{~cm}$ in our culturing system which acted as an oxygen

106 barrier.

107 In the human body, mature P. falciparum parasites (trophozoites and schizonts)

108 exhibit a preference for the venules where relatively high $\mathrm{O}_{2}$ levels are present (Milner

109 et al., 2015), Research on the effect of oxygen on the growth of Babesia spp has not

110 been reported in vitro or in vivo. In contrast, our in vitro results showed $B$. duncani

111 schizonts were more likely arrested in anaerobic and microaerobic conditions than 
112 ambient oxygen rich condition (Figure 2). Specifically, even though B. duncani did not

113 grow in anaerobic container $\left(0.7-1 \% \mathrm{O}_{2}\right)$, the ratio of schizonts in infected erythrocytes

114 soared immediately at the beginning and could finally reach to nearly $70 \%$ schizonts

115 before all parasites died out (Figure 2). The ratios of schizonts were increasing along

116 with increasing parasitemia and oxygen consumption in both microaerobic and aerobic

117 conditions, but the ratios of schizonts cultured in microaerobic condition were higher

118 than that cultured in aerobic condition at each day and entered plateau one day earlier.

119 This indicates that low $\mathrm{O}_{2}$ is able to extend the duration of $B$. duncani schizonts in vitro

120 or prevent schizonts from lysing erythrocytes to generate merozoites, which could also

121 happen in late culture phase. In contrast, Briolant et al. found that $P$. falciparum clones

$1223 \mathrm{D} 7$ and $\mathrm{W} 2$ had higher percentages of schizonts when inoculated in $21 \% \mathrm{O}_{2}$ than that

123 inoculated in $5 \% \mathrm{O}_{2}$, even though the growth of these two $P$. falciparum clones did not

124 show significant difference in $21 \%$ and $5 \% \mathrm{O}_{2}$ (Briolant et al., 2007).

125 Slight variations of in vitro culture environments have demonstrated significant

126 impact on the conclusions of P. falciparum drug resistance (Duffy and Avery, 2018). In

127 our study, we found that B. duncani cultured in microaerobic condition showed

128 significantly higher susceptibility to four anti-parasite drugs atovaquone,

129 pyrimethamine, quinine, and chloroquine at $0.25-50 \mu \mathrm{M}$ than that cultured in aerobic

130 condition, except that atovaquone had almost $100 \%$ inhibitory effects against $B$.

$131 d u n c a n i$ at $2.5,25$, and $50 \mu \mathrm{M}$ in both aerobic and microaerobic conditions (Figure 3 ).

132 The most variation of inhibitory effects occurred in chloroquine exposure, which had

$13365-100 \%$ inhibitory effect on $B$. duncani at 0.25 and $2.5 \mu \mathrm{M}$ in microaerobic condition,

134 but the effect was only 9-34\% at the same drug concentrations in aerobic condition

135 (Figure 3). As mentioned above, given P. falciparum 3D7 and W2 have the opposite

136 manifestation of ratios of schizonts in different $\mathrm{O}_{2}$ levels compared to $B$. duncani in this 
137 study, it is not surprising that $P$. falciparum 3D7 and W2 were more susceptible to

138 chloroquine in aerobic condition than that in microaerobic condition (Briolant et al.,

139 2007), which is dissimilar to the chloroquine susceptibility of $B$. duncani as shown in

140 our study. We speculated that these different consequences could result from the same

141 reason that schizonts are more susceptible to chloroquine due to chloroquine

142 accumulation might be much greater in schizont stage than in ring stage (Yayon et al.,

143 1983). Besides, some compounds have shown various activities against highly

144 synchronous age defined P. falciparum parasites (Duffy and Avery, 2017), however, to

145 date there is no way to study drug susceptibility of Babesia spp at a certain life cycle

146 stage due to the lack of effective synchronization of Babesia spp. Drug susceptibilities

147 of other Babesia spp impacted by $\mathrm{O}_{2}$ levels need to be studied in the future, and

148 culturing conditions of Babesia spp should be reestablished for generating more

149 comparable and reliable results in drug research and the relevant conditions that reflect

150 in vivo drug treatment effect should be determined to identify more effective drugs.

\section{Reference}

153 CDC - Babesiosis - Data \& Statistics Data. Available online:

154 https://www.cdc.gov/parasites/babesiosis/data-statistics/index.html (accessed on 1 June 155 2020).

156 Abraham, A., Brasov, I., Thekkiniath, J., Kilian, N., Lawres, L., Gao, R., DeBus, K., He,

157 L., Yu, X., Zhu, G., Graham, M.M., Liu, X., Molestina, R., Ben Mamoun, C., 2018.

158 Establishment of a continuous in vitro culture of Babesia duncani in human

159 erythrocytes reveals unusually high tolerance to recommended therapies. J Biol Chem

160 293, 19974-19981.

161 Branco, A., Francisco, D., Hanscheid, T., 2018. Is There a 'Normal' Oxygen

162 Concentration for in vitro Plasmodium Cultures? Trends in Parasitology 34, 811-812.

163 Brasseur, P., Lecoublet, S., Kapel, N., Favennec, L., Ballet, J.J., 1998. In vitro

164 evaluation of drug susceptibilities of Babesia divergens isolates. Antimicrob Agents

165 Chemother 42, 818-820.

166 Briolant, S., Parola, P., Fusai, T., Madamet-Torrentino, M., Baret, E., Mosnier, J.,

167 Delmont, J.P., Parzy, D., Minodier, P., Rogier, C., Pradines, B., 2007. Influence of

168 oxygen on asexual blood cycle and susceptibility of Plasmodium falciparum to

169 chloroquine: requirement of a standardized in vitro assay. Malar J 6, 44. 
170 Duffy, S., Avery, V.M., 2017. Plasmodium falciparum in vitro continuous culture 171 conditions: A comparison of parasite susceptibility and tolerance to anti-malarial drugs 172 throughout the asexual intra-erythrocytic life cycle. Int J Parasitol Drugs Drug Resist 7, 173 295-302.

174 Duffy, S., Avery, V.M., 2018. Routine In Vitro Culture of Plasmodium falciparum:

175 Experimental Consequences? Trends Parasitol 34, 564-575.

176 Jensen, J.B., Trager, W., 1977. Plasmodium falciparum in culture: use of outdated

177 erthrocytes and description of the candle jar method. J Parasitol 63, 883-886.

178 Kjemtrup, A.M., Conrad, P.A., 2000. Human babesiosis: an emerging tick-borne

179 disease. Int J Parasitol 30, 1323-1337.

180 Levy, M.G., Ristic, M., 1980. Babesia bovis: continuous cultivation in a

181 microaerophilous stationary phase culture. Science 207, 1218-1220.

182 McCormack, K.A., Alhaboubi, A., Pollard, D.A., Fuller, L., Holman, P.J., 2019. In vitro 183 cultivation of Babesia duncani (Apicomplexa: Babesiidae), a zoonotic hemoprotozoan, 184 using infected blood from Syrian hamsters (Mesocricetus auratus). Parasitol Res 118, 185 2409-2417.

186 Milner, D.A., Jr., Lee, J.J., Frantzreb, C., Whitten, R.O., Kamiza, S., Carr, R.A., 187 Pradham, A., Factor, R.E., Playforth, K., Liomba, G., Dzamalala, C., Seydel, K.B., 188 Molyneux, M.E., Taylor, T.E., 2015. Quantitative Assessment of Multiorgan

189 Sequestration of Parasites in Fatal Pediatric Cerebral Malaria. J Infect Dis 212, 1317-

1901321.

191 Nehrbass-Stuedli, A., Boykin, D., Tidwell, R.R., Brun, R., 2011. Novel diamidines with

192 activity against Babesia divergens in vitro and Babesia microti in vivo. Antimicrob

193 Agents Chemother 55, 3439-3445.

194 Timms, P., 1980. Short term cultivation of Babesia species. Res Vet Sci 29, 102-104.

195 Vega, C.A., Buening, G.M., Green, T.J., Carson, C.A., 1985. In vitro cultivation of

196 Babesia bigemina. Am J Vet Res 46, 416-420.

197 Yayon, A., Vandewaa, J.A., Yayon, M., Geary, T.G., Jensen, J.B., 1983. Stage-

198 Dependent Effects of Chloroquine on Plasmodium-Falciparum Invitro. J Protozool 30,

199 642-647.

200 Zhang, Y., Bai, C., Shi, W., Alvarez-Manzo, H., Zhang, Y., 2020. Identification of

201 Essential Oils Including Garlic Oil and Black Pepper Oil with High Activity against

202 Babesia duncani. Pathogens 9. 
206 Figure 1. (A) B. duncani-infected hamster erythrocytes observed at 1,000x

207 magnification in young (2 days old; left) and old (5-6 days old; right) cultures with

208 parasitemia of approximate $1.5 \%$ and $40 \%$, respectively. The growth of parasites started

209 from $0.5 \%$ parasitemia; (B) B. duncani schizonts appearing as budding four daughter

210 merozoites in erythrocytes displayed several different scattered distributions. B1,

211 Maltese Cross tetrad; B2, four daughter merozoites connected in pairs; B3, three

212 daughter merozoites assembled with one in isolation; B4, the moment of matured

213 merozoites bursting erythrocyte; B5, two schizonts in one erythrocyte; B6, a schizont

214 accompanied with ring form trophozoite;

215 Figure 2. Growth (left) and percentage of schizonts (right) of B. duncani cultures

216 incubated in aerobic, microaerobic, and anaerobic environments;

217 Figure 3. B. duncani incubated in aerobic and microaerobic conditions with a starting

$2181.5 \%$ parasitemia and $2.5 \%$ hematocrit ( 2 days old culture) treated with atovaquone,

219 pyrimethamine, quinine, and chloroquine at $0.25,2.5,25,50 \mu \mathrm{M}$ after 3 days exposure. 
bioRxiv preprint doi: https://doi.org/10.1101/2021.01.11.426147; this version posted January 12, 2021. The copyright holder for this preprint (which was not certified by peer review) is the author/funder. All rights reserved. No reuse allowed without permission.

$221 \quad$ Figure 1

A
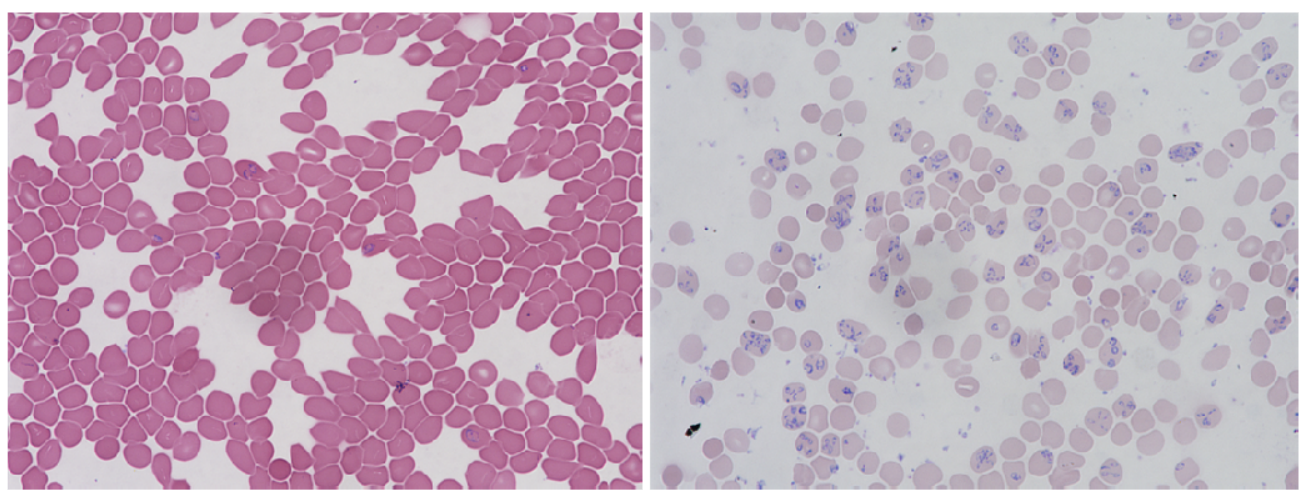

B

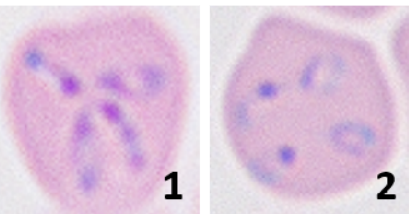

222
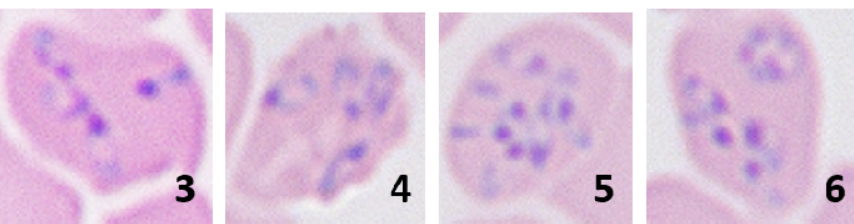
224 Figure 2
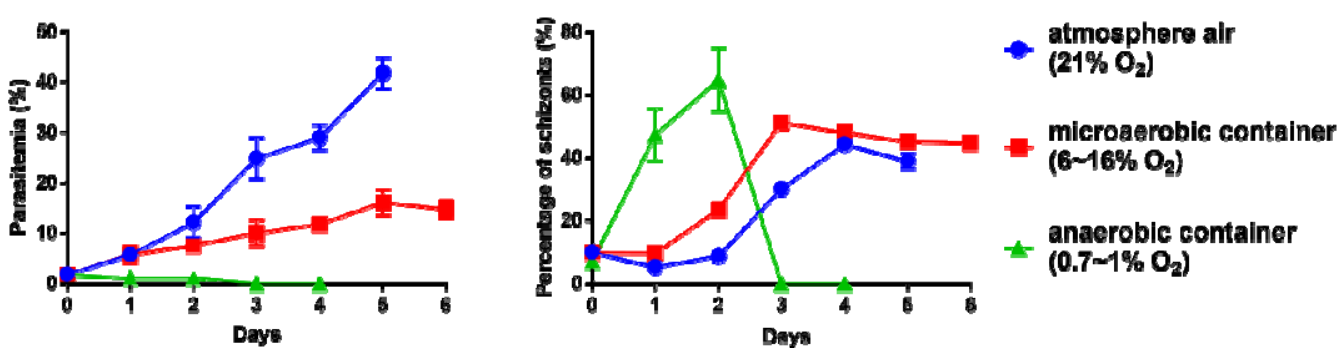

225 
Figure 3
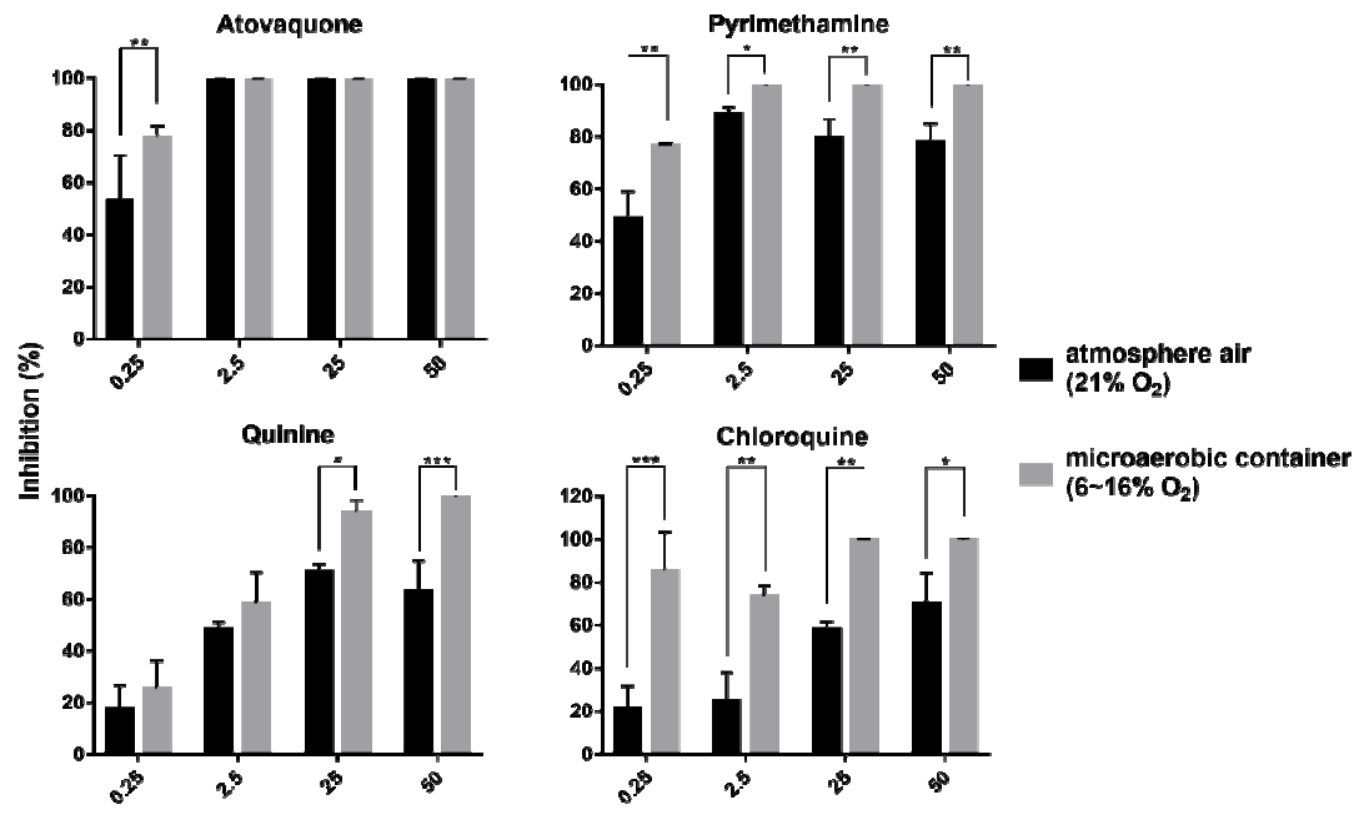

Drug concentrations $(\mu \mathrm{M})$ 

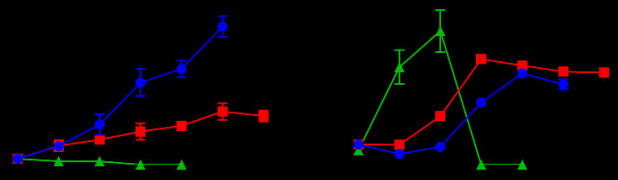

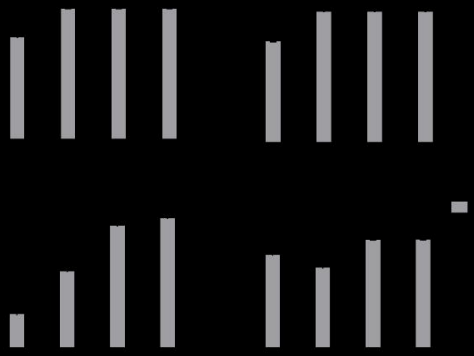